\title{
Association Study among Comethylation Modules, Genetic Polymorphisms and Clinical Features in Mexican Teenagers with Eating Disorders: Preliminary Results
}

\author{
Germán Alberto Nolasco-Rosales ${ }^{1}{ }^{\circledR}$, José Jaime Martínez-Magaña ${ }^{2}{ }^{\circledR}$, Isela Esther Juárez-Rojop ${ }^{1}$, \\ Thelma Beatriz González-Castro $\left.{ }^{3}{ }^{(}\right)$, Carlos Alfonso Tovilla-Zarate ${ }^{4}{ }^{(}$, Ana Rosa García ${ }^{5}$, Emmanuel Sarmiento ${ }^{5}$, \\ David Ruiz-Ramos ${ }^{1}{ }^{\mathbb{D}}$, Alma Delia Genis-Mendoza ${ }^{2, *}$ and Humberto Nicolini ${ }^{2, * \mathbb{C}}$
}

1 Biomedical Postgraduate Program, Academic Division of Health Sciences, Juárez Autonomous University of Tabasco, Villahermosa 86000, Mexico; ganr_1277@live.com.mx (G.A.N.-R.); iselajuarezrojop@hotmail.com (I.E.J.-R.); daruiz_914@hotmail.com (D.R.-R.)

2 Genomics of Psychiatric and Neurodegenerative Diseases Laboratory, National Institute of Genomic Medicine (INMEGEN), Mexico City 01090, Mexico; martinezmaganajjaime@gmail.com

3 Genomics Laboratory, Academic Division Jalpa de Mendez, Juárez Autonomous University of Tabasco, Jalpa de Mendez 86200, Mexico; thelma.glez.castro@gmail.com

check for

updates

Citation: Nolasco-Rosales, G.A.; Martínez-Magaña, J.J.; Juárez-Rojop, I.E.; González-Castro, T.B.;

Tovilla-Zarate, C.A.; García, A.R.; Sarmiento, E.; Ruiz-Ramos, D.; Genis-Mendoza, A.D.; Nicolini, H. Association Study among Comethylation Modules, Genetic Polymorphisms and Clinical Features in Mexican Teenagers with Eating Disorders: Preliminary Results. Nutrients 2021, 13, 3210. https:// doi.org/10.3390/nu13093210

Academic Editors: Daniel-Antonio de Luis Roman and Ana B. Crujeiras

Received: 9 July 2021

Accepted: 7 September 2021

Published: 15 September 2021

Publisher's Note: MDPI stays neutral with regard to jurisdictional claims in published maps and institutional affiliations.

Copyright: (c) 2021 by the authors. Licensee MDPI, Basel, Switzerland. This article is an open access article distributed under the terms and conditions of the Creative Commons Attribution (CC BY) license (https:/ / creativecommons.org/licenses/by/ $4.0 /)$.
4 Genomics Laboratory, Comalcalco Multidisciplinary Academic Division, Juárez Autonomous University of Tabasco, Villahermosa 86000, Mexico; alfonso_tovillaz@yahoo.com.mx

5 Children's Psychiatric Hospital “Dr. Juan N. Navarro”, Mexico City 01090, Mexico; anarosagarciab@gmail.com (A.R.G.); emmanuelsarmientoh@hotmail.com (E.S.)

* Correspondence: adgenis@inmegen.gob.mx (A.D.G.-M.); hnicolini@inmegen.gob.mx (H.N.); Tel.: +52-(53)-501900 (ext. 1196/1197) (A.D.G.-M. \& H.N.)

Abstract: Eating disorders are psychiatric disorders characterized by disturbed eating behaviors. They have a complex etiology in which genetic and environmental factors interact. Analyzing geneenvironment interactions could help us to identify the mechanisms involved in the etiology of such conditions. For example, comethylation module analysis could detect the small effects of epigenetic interactions, reflecting the influence of environmental factors. We used MethylationEPIC and Psycharray microarrays to determine DNA methylation levels and genotype from 63 teenagers with eating disorders. We identified 11 comethylation modules in WGCNA (Weighted Gene Correlation Network Analysis) and correlated them with single nucleotide polymorphisms (SNP) and clinical features in our subjects. Two comethylation modules correlated with clinical features (BMI and height) in our sample and with SNPs associated with these phenotypes. One of these comethylation modules (yellow) correlated with BMI and rs10494217 polymorphism (associated with waist-hip ratio). Another module (black) was correlated with height, rs9349206, rs11761528, and rs17726787 SNPs; these polymorphisms were associated with height in previous GWAS. Our data suggest that genetic variations could alter epigenetics, and that these perturbations could be reflected as variations in clinical features.

Keywords: comethylation modules; genetic polymorphisms; eating disorders; WGCNA

\section{Introduction}

Eating disorders (EDs) are severe psychiatric disorders characterized by disturbances of eating behavior, affecting the health and quality of life of individuals. These disorders have an early teenage onset and a hereditary component [1]. EDs have a complex etiology in which genetic and environmental factors interact [2]. Genome-wide association studies (GWAS) and other genetic studies have revealed loci and single nucleotide polymorphisms (SNP) associated with ED [1,3,4]. The clinical characteristics of EDs have been associated in genetic studies. In this sense, significant genetic correlations have been reported in anorexia nervosa with psychiatric disorders, physical activity and metabolic, lipid and 
anthropometric traits [5]. In addition, genetic associations between ED and substance use have been described [6]. Additionally, the genetic-environment relationship in ED has been studied through DNA methylation, reporting perturbations in the methylation levels of some genes (DRD2, SLC6A3, POMC, OXTR, among others) $[2,7,8]$. However, genes do not function alone: on average, each gene is estimated to interact with another four or eight genes, and to be involved with 10 biological functions. Furthermore, recent studies suggest that gene networks provide the potential to identify hundreds of disease-related genes [9]. Analyzing gene-environment interactions in EDs could help us to identify the mechanisms involved in their etiology. Nowadays, new technologies evaluating thousands of genes apply statistic approaches that integrate different information sources from gene interactions (e.g., comethylation module construction) [10]. Comethylation modules are clusters of highly interconnected CPG sites. These modules are detected through the construction of a correlation network. Correlation networks are used to analyze large, high-dimensional data sets. These correlation networks are constructed on the basis of correlations among quantitative measurements (e.g., gene expression profiles, methylation levels) [11]. Comethylation modules are formed by using methylation data as quantitative measurements of gene-environment interactions [10]. Additionally, comethylation modules alleviate various testing problems which are inherent to microarray data analyses, and have been found to be useful for describing pairwise relationships among methylated genes [9-11]. In brief, comethylation modules (1) consider all genes as interconnected, (2) identify groups of CpG sites with similar methylation levels, (3) increase statistical power, and (4) detect small effects of epigenetic interactions [9-11]. Thus, evaluating correlations among genetic factors, comethylation modules, and clinical features in EDs could be a means by which to identify biological markers in such disorders. The objective of the present study was to detect comethylation modules from DNA methylation samples from children and teenagers with an ED, and to correlate these modules with clinical features and genetic variability.

\section{Materials and Methods}

\subsection{Study Population}

We included 63 subjects diagnosed with anorexia nervosa (AN), bulimia nervosa, (BN) or binge eating disorder (BED) using DSM 5 criteria [12]. Individuals were recruited in the outpatient center of the Children's Psychiatric Hospital "Dr. Juan N. Navarro" from May 2014 to August 2016. Inclusion criteria were subjects with at least three generations of Mexican lineage, 12-18 years of age, and individuals not using psychotropic or psychoactive drugs. The clinical features of the sample are descripted in Table 1.

Table 1. Clinical features of study population.

\begin{tabular}{cc}
\hline Features & Sample $(\boldsymbol{n}=\mathbf{5 0})$ \\
\hline Age (years) & $13.98 \pm 1.74$ \\
Gender & $13(26)$ \\
Male & $37(74)$ \\
Female & $1.03 \pm 0.97$ \\
Body Mass Index (BMI) zscore & $1(2)$ \\
BMI classification & $20(40)$ \\
Underweight & $11(22)$ \\
Normal weight & $18(36)$ \\
Overweight & \\
Obesity & $17(34)$ \\
Diagnosis & $22(44)$ \\
Binge eating disorder & $11(22)$ \\
Bulimia nervosa & \\
Anorexia nervosa & $46(92)$ \\
Comorbidities & \\
Any &
\end{tabular}


Table 1. Cont.

\begin{tabular}{cc}
\hline Features & Sample $(\boldsymbol{n}=\mathbf{5 0})$ \\
\hline Major depressive disorder & $21(42)$ \\
Suicide behavior & $16(32)$ \\
Dysthymia disorder & $18(36)$ \\
Attention-Deficit/Hyperactivity Disorder & $15(30)$ \\
Generalized Anxiety Disorder & $10(20)$ \\
Oppositional Defiant Disorder & $6(12)$ \\
Conduct Disorder & $5(10)$ \\
Psychotic Disorder & $5(10)$ \\
Eating Attitudes & \\
Fear of gain weight & $35(70)$ \\
Binge & $34(68)$ \\
Restriction & $24(48)$ \\
Vomit & $21(42)$ \\
Other behaviors & $10(20)$
\end{tabular}

Features of subjects who satisfied inclusion criteria. Continuous variables are expressed as mean \pm standard deviation. Categorical variables are expressed as $n(\%)$.

This study followed the principles of the Declaration of Helsinki. Sample recollection and processing were approved by the Ethics Committee of the Children's Psychiatric Hospital “Dr. Juan N. Navarro" with approval No. II3/01/0913 (11 October 2017), and by the Ethics Committee of the National Institute of Genomic Medicine (INMEGEN) with approval No. 06/2018/I.

\subsection{Evaluation Instruments}

BED was screened with the QEWP-R (Questionnaire on Eating and Weight PatternRevised) [13]. AN was screened with EAT-26 (Eating Attitudes Test) [14]. We evaluated the presence of psychiatric comorbidity with the Spanish version of MINI Kid (Mini International Neuropsychiatric Interview for Children and Adolescent) [15]. A pedopsychiatrist performed all ED diagnoses.

\subsection{DNA Extraction and Microarray Analysis}

After diagnostic evaluation of each individual, a blood sample was collected using an EDTA tube; DNA was subsequently extracted from this sample. We used the salting-out method from the Gentra Puregene Blood (Qiagen, Germantown, MD, USA) commercial kit. DNA extraction quality and integrity were evaluated by analysis with a NanoDrop spectrophotometer (Thermofisher, Waltham, MA, USA) and $2 \%$ agarose gel. DNA samples met the following quality criteria: visible genomic DNA band, 230/260 and 260/230 ratios $>1.8$, concentration $>50 \mathrm{ng} / \mu \mathrm{L}$, and no signs of DNA degradation. For genotypification, we hybridized DNA with commercial microarray Infinium Psycharray Beadchip (Illumina, San Diego, CA, USA). For methylation analysis, DNA was bisulfite converted using an EZ DNA Methylation Kit (Zymo Research, Irvine, CA, USA). Converted DNA was hybridized with the Infinium MethylationEPIC BeadChip (Illumina, San Diego, CA, USA). Each microarray was processed in the Microarray and Expression Unit of the National Institute of Genomic Medicine.

\subsection{Quality Control of Genotypification Data}

We transformed fluorescence intensities from the Psycharray into genotypes using the GenomeStudio (v. 2.0) software, and quality control was done with the PLINK (v. 1.9) toolset [16]. We eliminated: (1) SNPs with less than 95\% genotype calls, (2) individuals with less than 95\% genotype calls, (3) individuals with sex discrepancy, (4) SNPs located in chromosomes $\mathrm{X}$ and $\mathrm{Y}$, (5) SNPs with less than 0.05 minor allele frequency (MAF), (6) SNPs deviating from Hardy-Weinberger equilibrium $\left(p<1 \times 10^{-6}\right)$, and (7) SNPs with A/T and C/G alleles. Subsequently, filtrated data were exported to the R (v. 4.0) software [17], and 
we removed SNPs with missing data and SNPs without homozygous individuals to minor alleles. Only 193,314 SNPs passed the quality control.

\subsection{Quality Control of DNA Methylation Data}

The fluorescence intensities of the MethylationEPIC microarray were transformed into idat files, which were filtered with ChAMP pipeline (v.2.18) [18] for R (v. 4.0) software. Quality control removed: (1) probes with detection $p$-value $>0.01,(2)$ probes with $<3$ beads in at least $5 \%$ of samples per probe, (3) non-CpG probes, (4) multihit probes, (5) probes located in chromosome $X$ and $Y$, and (6) individuals with sex discrepancy in their genotypification data. We converted filtered methylation data into $\beta$-values, which were normalized using the BMIQ (Beta-Mixture Quantile Normalization) method [19]. Afterwards, we evaluated the presence of the batch effect with a singular value decomposition (SVD) method. We preserved CpG sites with standard deviation (SD) $>0.05$, keeping 105,393 sites. Likewise, we made many cut points in $\operatorname{SD}(0.05,0.06,0.07,0.08,0.09,0.10,0.15,0.20)$ to find an optimal point for the construction of comethylation modules.

\subsection{Comethylation Modules Construction}

In order to identify comethylation modules, we processed methylation values with the WGCNA (Weighted correlation network analysis) package [11,20] (R software). Later, we applied the means method to achieve hierarchical clustering, and eliminated individuals with atypical samples. For the final analysis, we only considered 50 subjects. Furthermore, an analysis of network topology was used to determine a soft-thresholding power less than 20 with suitable independence $(>0.8)$ and mean connectivity $(<1000)$. The CpG sites with SD $>0.06$ in their methylation values had the best network topology. We applied blockwiseModules function (WGCNA package $[11,20]$ ) to detect comethylation modules, using a minimum module size of 175 and a threshold of 20. In a subsequent analysis, we discarded the CPG sites clustered in the grey module, and identified a new set of modules. The 11 constructed comethylation modules included 11,418 CpG sites. Each module was automatically assigned a color by WGCNA, indicating its size. The grey module was discarded from further analysis as it groups unassigned CpG sites to other modules; thus, these sites are unrelated.

\subsection{Enrichment Analysis of Modules}

The CpG sites inside modules were annotated using IlluminaHumanMethylationEPICanno.ilm10b4.hg19 package [21]. Genes of CpG sites were extracted and enriched using the WebGestalt online tool [22]. We accomplished the enrichments with OverRepresentation Analysis of KEGG Database (Kyoto Encyclopedia of Genes and Genomes) [23]; this was considered to be significant with an adjusted $p$-value by FDR $\leq 0.05$.

\subsection{Correlation of Comethylation Modules with Clinical Features and SNPS}

The eigengene of each comethylation module was correlated with clinical data and SNPs using Pearson's correlations. We calculated the $\mathrm{R}^{2}$ and $p$ values with cor and corPvalueStudent functions and set the significant value $p<5 \times 10^{-3}$ for clinical data and $p<5$ $\times 10^{-8}$ for SNPs. In order to find associations between SNPs and phenotypes, we used the PheWAS tool on the GWAS Atlas website [24]. Associations were considered to be significant for any phenotype with a $p<1 \times 10^{-10}$.

\section{Results}

\subsection{Description of Comethylation Modules}

There were 11 modules in our study. Modules were turquoise (5073 sties), blue (2928 sites), brown (193 sites), yellow (166 sites), green (151 sites), red (150 sites), black (148 sites), pink (145 sites), magenta (135 sites), purple (111 sites), and grey (2218 sites). The CpG sites of these modules were located in 4005 genes. According to relative position to gene, the gene body was the most common annotated location, ranging from 40 sites 
(56.34\%) in the purple module to 2430 sites $(69.27 \%)$ in the turquoise module. Table 2 shows the details of functional annotation of $\mathrm{CpG}$ sites from comethylation modules regarding gene location.

Table 2. Comethylation module CpG sites classification.

\begin{tabular}{cccccccc}
\hline Module & TSS1500 & TSS200 & $\mathbf{5}^{\prime}$ UTR & Body & 1stExon & ExonBnd & $\mathbf{3}^{\prime}$ UTR \\
\hline Turquoise & $359(10.23)$ & $97(2.77)$ & $436(12.43)$ & $2430(69.27)$ & $33(0.94)$ & $37(1.05)$ & $116(3.31)$ \\
Blue & $158(7.85)$ & $75(3.72)$ & $309(15.34)$ & $1366(67.83)$ & $23(1.14)$ & $16(0.79)$ & $67(3.33)$ \\
Brown & $14(10.77)$ & $4(3.08)$ & $23(17.69)$ & $78(60.00)$ & $2(1.54)$ & $3(2.31)$ & $6(4.62)$ \\
Yellow & $13(12.15)$ & $6(5.61)$ & $13(12.15)$ & $69(64.49)$ & $2(1.87)$ & $0(0)$ & $4(3.74)$ \\
Green & $11(11.22)$ & $3(3.06)$ & $13(13.27)$ & $68(69.39)$ & $1(1.02)$ & $1(1.02)$ & $1(1.02)$ \\
Red & $20(18.52)$ & $1(0.93)$ & $15(13.89)$ & $66(61.11)$ & $2(1.85)$ & $1(0.93)$ & $3(2.78)$ \\
Black & $11(11.22)$ & $2(2.04)$ & $9(9.18)$ & $66(67.35)$ & $2(2.04)$ & $2(2.04)$ & $6(6.12)$ \\
Pink & $13(13.00)$ & $2(2.00)$ & $9(9.00)$ & $71(71.00)$ & $2(2.00)$ & $1(1.00)$ & $2(2.00)$ \\
Magenta & $10(11.36)$ & $4(4.55)$ & $12(13.64)$ & $56(63.64)$ & $1(1.14)$ & $0(0)$ & $5(5.68)$ \\
Purple & $8(11.27)$ & $5(7.04)$ & $8(11.27)$ & $40(56.34)$ & $1(1.41)$ & $2(2.82)$ & $7(9.86)$ \\
\hline
\end{tabular}

CpG sites were annotated with the IlluminaHumanMethylationEPICanno.ilm10b4.hg19 [21] package. Data expressed as $n$ of sites, (\%) by rows. TSS: Transcription Start Site. UTR: Untranslated Region. ExonBnd: Exon Boundaries.

Concerning the distribution of $\mathrm{CpG}$ sites with respect to $\mathrm{CpG}$ islands, a majority of comethylation modules corresponded to Open Sea (71.11-84.56\%, 85-4207 sites); on the other hand, the purple comethylation module had a high percentage of CpG sites annotated on islands (15.32\%, 17 sites) (Table 3). CpG sites in comethylation modules were heterogeneously distributed among chromosomes (Table S1). Additionally, we observed two groups of comethylation modules given the methylation levels from beta values. One group had partially methylated values $(0.2<\beta$ value $<0.8)$ (turquoise, blue and purple), while the other group had hypermethylated values ( $\beta$ value $\geq 0.8$ ) (brown, yellow, green, red, black, pink and magenta) (Table S2).

Table 3. CpG site position with respect to $\mathrm{CpG}$ islands.

\begin{tabular}{|c|c|c|c|c|c|c|}
\hline Module & OpenSea & Island & N Shore & S Shore & N Shelf & S Shelf \\
\hline Turquoise & 4207 (82.93) & $13(0.26)$ & $247(4.87)$ & $212(4.18)$ & $202(3.98)$ & $192(3.78)$ \\
\hline Blue & $2476(84.56)$ & $15(0.51)$ & $128(4.37)$ & $83(2.83)$ & $104(3.55)$ & $122(4.17)$ \\
\hline Brown & 153 (79.27) & $6(3.11)$ & $9(4.66)$ & $17(8.81)$ & $4(2.07)$ & $4(2.07)$ \\
\hline Yellow & $125(75.30)$ & $1(0.60)$ & $14(8.43)$ & $10(6.02)$ & $12(7.23)$ & $4(2.41)$ \\
\hline Green & $114(75.50)$ & $5(3.31)$ & $10(6.62)$ & $8(5.30)$ & $9(5.96)$ & $5(3.31)$ \\
\hline Red & $121(80.67)$ & $1(0.67)$ & $6(4.00)$ & $2(1.33)$ & $8(5.33)$ & $12(8.00)$ \\
\hline Black & 115 (77.70) & $1(0.68)$ & $7(4.73)$ & $6(4.05)$ & $11(7.43)$ & $8(5.41)$ \\
\hline Pink & $114(78.62)$ & $6(4.14)$ & $7(4.83)$ & $7(4.83)$ & $8(5.52)$ & $3(2.07)$ \\
\hline Magenta & $96(71.11)$ & $6(4.44)$ & $9(6.67)$ & $9(6.67)$ & $8(5.93)$ & 7 (5.19) \\
\hline Purple & 85 (76.58) & $17(15.32)$ & $4(3.60)$ & $3(2.70)$ & $1(0.90)$ & $1(0.90)$ \\
\hline Total & $7606(82.67)$ & $71(0.77)$ & $441(4.79)$ & $357(3.88)$ & 367 (3.99) & $358(3.89)$ \\
\hline
\end{tabular}

CpG sites were annotated with the IlluminaHumanMethylationEPICanno.ilm10b4.hg19 [21] package. Data expressed in $n$ of sites, (\%) by rows.

\subsection{Enriched Pathways on Each Module}

We found significant enriched pathways of genes annotated on the CpG sites from turquoise and blue modules (Table S3). Genes in the turquoise module were enriched for the longevity regulating pathway (adjusted $p$ value $=0.0047$ ), $\mathrm{GnRH}$ (Gonadotropin-releasing hormone) signaling pathway (adjusted $p$ value $=0.0042$ ), glioma (adjusted $p$ value $=0.0126$ ), cholinergic synapse (adjusted $p$ value $=0.0091$ ), human cytomegalovirus infection (adjusted $p$ value $=0.0126$ ), and endocytosis (adjusted $p$ value $=0.0126$ ). Genes from blue comethylation module were enriched in pathways for Th1 and Th2 cell differentiation (adjusted $p$ value $=6.8672 \times 10^{-7}$ ), allograft rejection (adjusted $p$ value $=0.0185$ ), endometrial cancer (adjusted $p$ value $=0.0111$ ), and TNF signaling pathway (adjusted $p$ value $=0.0007$ ). Another enrichment pathways within the same module included the AGE-RAGE signaling 
pathway in diabetic complications (adjusted $p$ value $=0.0033$ ), phosphatidylinositol signaling system (adjusted $p$ value $=0.0074$ ), glioma (adjusted $p$ value $=0.0365$ ), longevity regulating pathway (adjusted $p$ value $=0.0325$ ), human cytomegalovirus infection (adjusted $p$ value $=0.0008$ ), and focal adhesion (adjusted $p$ value $=0.0039$ ).

\subsection{Correlations of Modules with Clinical Features in Our Population}

In our results, seven clinical features and comorbidities correlated with different comethylation modules (Figure 1). The yellow comethylation module correlated with body mass index (BMI) zscore $\left(\mathrm{R}^{2}=0.47, p=0.0006\right)$, conduct disorder $\left(\mathrm{R}^{2}=-0.41, p=0.0030\right)$, and psychotic disorder $\left(\mathrm{R}^{2}=-0.45, p=0.0010\right)$. Meanwhile, the purple comethylation module correlated with gender $\left(R^{2}=-1, p<1 \times 10^{-50}\right)$, suicidal behavior $\left(R^{2}=0.41\right.$, $p=0.0030)$, and attention-deficit/hyperactivity disorder $\left(R^{2}=-0.59, p=6 \times 10^{-6}\right)$. Finally, the black module correlated with height $\left(\mathrm{R}^{2}=0.4, p=0.0040\right)$. Notably, clinical features did not correlate with more than one module at a time.

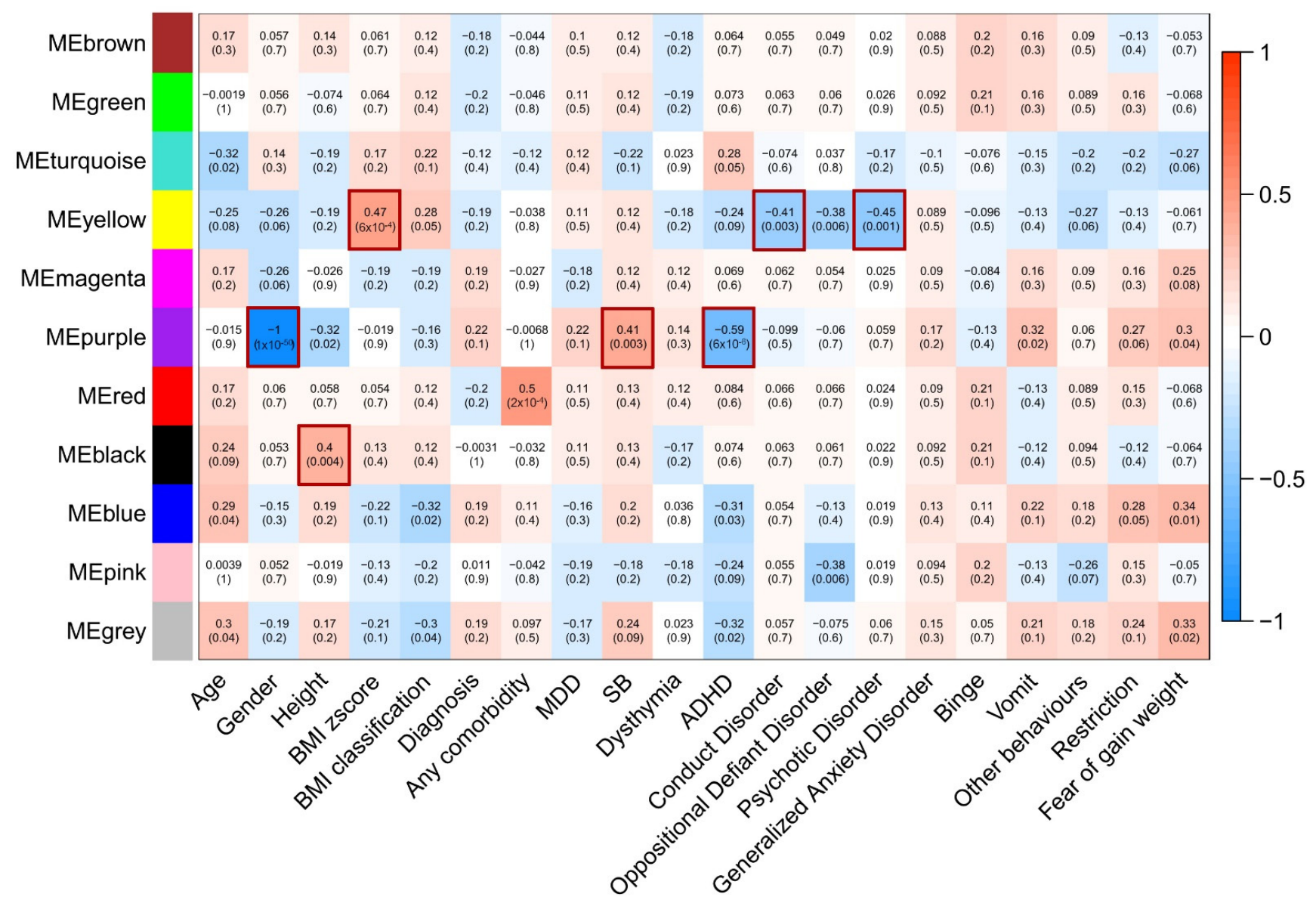

Figure 1. Correlations between modules and clinical features. MDD: major depressive disorder. SB: suicidal behavior. ADHD: attention deficit hyperactivity disorder. Red borders indicate significant correlations. Significance indicates $p$-values $<5 \times 10^{-3}$.

\subsection{Correlations of SNPs with Modules}

Seven comethylation modules had correlations with any SNP (brown, green, yellow, magenta, red, black and pink) (Table S4). SNPs were located mostly in intronic regions, ranging from $33.96 \%$ in the red module ( $18 \mathrm{SNPs}$ ) to $55.56 \%$ in the yellow module ( $15 \mathrm{SNPs}$ ). Another frequent location was intergenic regions, ranging from $14.81 \%$ in the yellow module (4 SNPs) to $31.71 \%$ in the black module (13 SNPs). The most frequent location in 
the green module was intergenic regions ( $28.13 \%$, $9 \mathrm{SNPs})$, followed by intronic regions $(21.88 \%, 7$ SNPs).

The most correlated SNPs $(89.95 \%, 206$ SNPs) were in nonprotein-coding transcript regions, while $10.05 \%$ ( $23 \mathrm{SNPs}$ ) were in protein-coding regions (synonymous and missense). Seventeen SNPs (7.42\%) were annotated as missense variants; the red and black modules had four missense SNPs each. Meanwhile, six correlated SNPs $(2.62 \%)$ were annotated as synonymous variants, with two SNPs per module (brown, yellow, and red). We observed 10 correlated SNPs (4.37\%) in regulatory regions, although no SNPs were found in the yellow comethylation module. The magenta comethylation module had no SNPs annotated in the upstream and downstream regions. Finally, correlated SNPs annotated in $3^{\prime}$ untranslated regions (UTR) were the least frequent (2 SNPs, $0.87 \%$ ), found within the magenta and red modules (Table 4).

Table 4. Annotations of SNPs correlated with modules.

\begin{tabular}{ccccccccc}
\hline & Brown & Green & Yellow & Magenta & Red & Black & Pink & Total \\
\hline 3'UTR & $0(0.00)$ & $0(0.00)$ & $0(0.00)$ & $1(5.88)$ & $1(1.89)$ & $0(0.00)$ & $0(0.00)$ & $2(0.89)$ \\
Downstream & $0(0.00)$ & $2(6.25)$ & $1(3.70)$ & $0(0.00)$ & $4(7.55)$ & $1(2.44)$ & $1(5.00)$ & $9(4)$ \\
Intergenic & $7(17.95)$ & $9(28.13)$ & $4(14.81)$ & $5(29.41)$ & $13(24.53)$ & $13(31.71)$ & $4(20.00)$ & $55(24.4)$ \\
Intron & $19(48.72)$ & $7(21.88)$ & $15(55.56)$ & $7(41.18)$ & $18(33.96)$ & $14(34.15)$ & $7(35.00)$ & $87(38.67)$ \\
Missense & $3(7.69)$ & $1(3.13)$ & $2(7.41)$ & $1(5.88)$ & $4(7.55)$ & $4(9.76)$ & $2(10.00)$ & $17(7.56)$ \\
Non coding & $3(7.69)$ & $8(28.13)$ & $2(7.41)$ & $1(5.88)$ & $6(13.21)$ & $5(14.64)$ & $3(20.00)$ & $28(12.44)$ \\
transcript & $1(2.56)$ & $3(9.38)$ & $0(0.00)$ & $2(11.76)$ & $2(3.77)$ & $1(2.44)$ & $1(5.00)$ & $10(4.44)$ \\
Regulatory & $2(5.13)$ & $0(0.00)$ & $2(7.41)$ & $0(0.00)$ & $2(3.77)$ & $0(0.00)$ & $0(0.00)$ & $6(2.67)$ \\
Synonymous & $4(10.26)$ & $1(3.13)$ & $1(3.70)$ & $0(0.00)$ & $2(3.77)$ & $2(4.88)$ & $1(5.00)$ & $11(4.89)$ \\
Upstream & & & & & & & & \\
\hline
\end{tabular}

dbSNP codes were annotated with InfiniumPsychArray-24v1-3_A1_b150_rsids file. Coding regions were annotated with Ensembl Variant Effect Predictor. Data expressed as $n$ of SNPs, (\%) by columns.

\subsection{Correlated SNP PheWAS}

Regarding clinical features, BMI, body fat, and height were the most frequent phenotypes associated with SNPs (Figure 2).

Concerning psychiatric disorders, we found several SNPs to be associated with three comethylation modules. The brown comethylation module had a SNP associated with depressive symptoms and neuroticism (rs4598994). Meanwhile, the pink comethylation module was associated with depressive affect (rs4800995); two SNPs were associated with schizophrenia (rs3129012 and rs356971) in the black comethylation module. Moreover, seven correlated SNPs with four comethylation modules were associated with autoimmune diseases. The magenta comethylation module was associated with rheumatoid arthritis and Crohn's disease (rs1893217). Likewise, the red (rs3095345) and pink (rs9267546 and rs9267547) comethylation modules were associated with rheumatoid arthritis and type 1 diabetes. The black module was associated with primary sclerosing cholangitis (rs3129012 and rs356971), autoimmune vitiligo, and systemic lupus erythematosus (rs356971). Finally, the yellow and black comethylation modules were correlated with clinical features in our population (BMI zscore, conduct disorder, psychotic disorder, and height); likewise, these comethylation modules were correlated with SNPs associated with similar phenotypes. Meanwhile, the yellow module correlated with one SNP (rs10494217) associated with the waist-hip ratio in PheWAS, and the black comethylation module correlated with three SNPs associated with height (rs9349206, rs11761528 and rs17726787). 


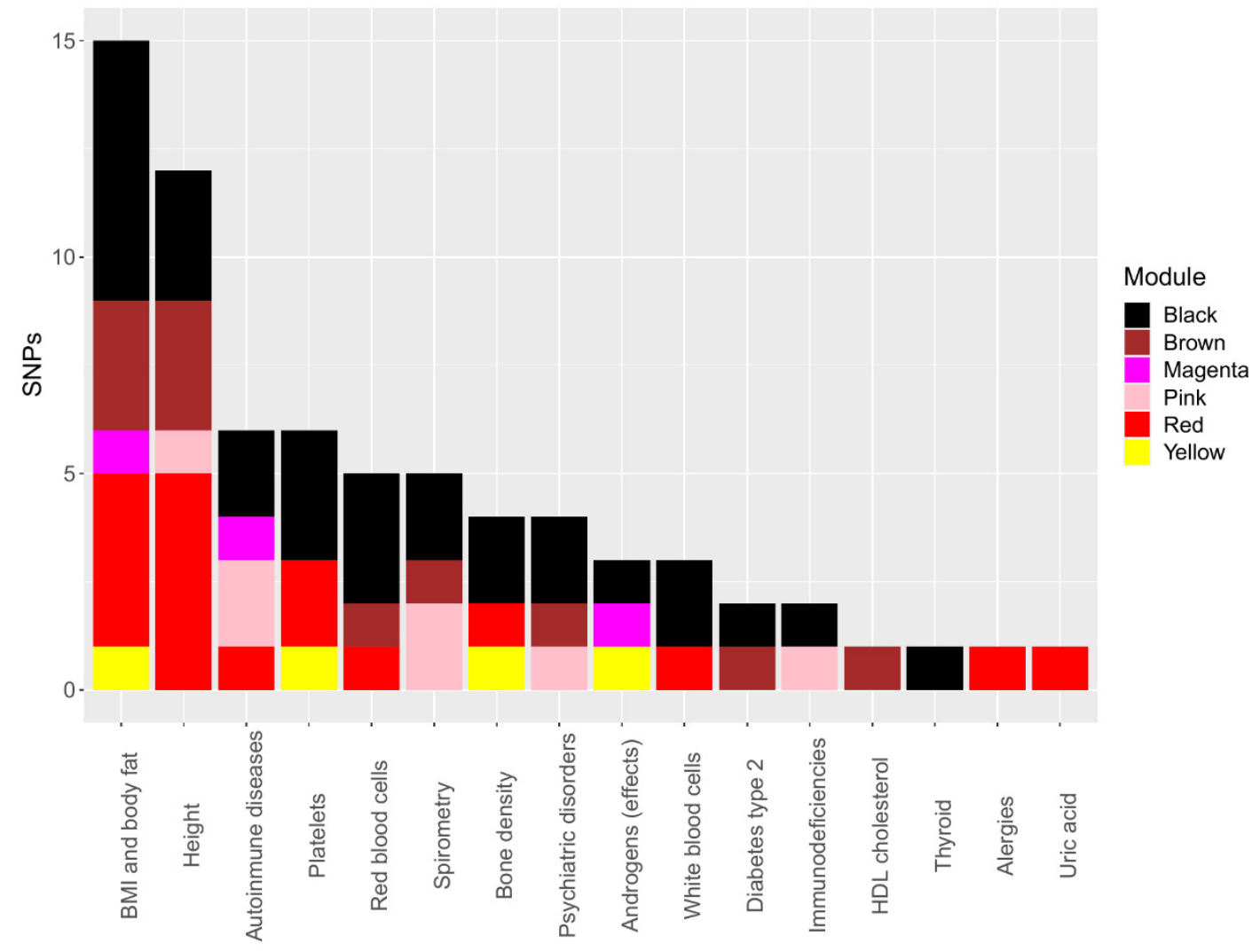

Figure 2. Phenotypes associated with correlated single nucleotide polymorphisms (SNP) in PheWAS.

\section{Discussion}

Several studies have evaluated the clinical features, genetic variants, and single DNA methylation sites involved in ED, but none has considered these factors together to date $[1,5,7]$. As such, little information is available about the integration of these different levels of biological information. As far as we know, this is the first study to integrate clinical features, genetic variants, and DNA methylation using a comethylation network analysis in teenagers with EDs.

BMI is an important clinical characteristic of the individuals diagnosed with ED, and it has a high impact on metabolic profiles [25]. Low BMI is a diagnosis criterion for anorexia nervosa [12]; on the other hand, bulimia nervosa and binge-eating disorder are related to risk of overweight/obesity [26]. In our analysis, the yellow module could be important in the changes in BMI found in individuals with EDs. The yellow module was correlated with BMI and rs10494217. This SNP is a missense genetic variant changing a histidine aminoacid for an asparagine in position 50 of TBX15 gene (p.His50Asn). Previously, GWAS associated rs10494217 with waist-hip index, a variable related to BMI [27]. The TBX15 gene is a member of the T-box gene family, i.e., transcriptional regulators which play an important role in the development of skeletal elements of limbs, vertebral column, and head, as well as other organs [28,29]. Likewise, this gene was reported as a regulator of metabolism of adipose tissue and muscle fibers, and shown to indirectly regulate body fat and BMI [30-32]. TBX15 is highly expressed in adipose tissue, and it binds to the promoter of PRDM16 gene. PRDM16 is essential for the browning of adipose tissue; reduced expression of its protein promotes obesity with high-fat diet and increases visceral fat [33]. As a missense variant, rs10494217 could reduce the binding of TBX15 protein to the promoter of PRDM16, and thus disturb adipose tissue function and alter BMI in individuals diagnosed with an ED. A possible alteration of PRDM16 expression could induce epigenetic reprogramming, as it is found in the yellow module in this study. CpG sites of the yellow module were enriched in alpha-linolenic acid metabolism (PLA2G4E and PLB1) and VEGF signaling pathway (AKT3, 
NFATC2, PLA2G4E and SHC2); both pathways are involved in adipose tissue function and BMI [34-37].

The black module could also be related to BMI in ED-diagnosed individuals. This module was correlated with rs11761528, a SNP associated with BMI and androsterone sulfate metabolism [27,38]. rs11761528 is an intronic polymorphism of the ZKSCAN5 gene. There is little information about ZKSCAN5 (zinc finger with KRAB and SCAN domains 5 ) and its mechanism; however, animal models suggest that this gene is correlated with adipocyte volume, systolic blood pressure, and cardiac mass [39]. Similarly, rs17726787 was previously associated with height and trunk fat-free mass in GWAS [24,40]. This SNP is an intronic variant of the CELF1 gene. Disturbances in gene expression of CELF1 are related with cardiopathies [41-43]. The black module was enriched in mTOR signaling pathway (IGF1R, LPIN1 and RPS6KA2), suggesting that genetic variations like rs11761528 and rs17726787 could alter the epigenetics of this pathway. The mTOR signaling pathway is essential for cardiac development [44,45]. Heart complications are frequent in anorexia nervosa patients, reaching $80 \%$ in some studies. Severe anorexia nervosa can change cardiac structure, although most structural abnormalities are reversible [46,47]. Nevertheless, there is a lack of analyses which explore the relationships between altered genes in the module, genetic variation, and cardiopathies in ED-diagnosed individuals.

Although schizophrenia was not correlated in our sample, we found genes and SNPs associated with the disorder. Schizophrenia and other psychiatric disorders are associated with anorexia nervosa [3,5]. Also, there is reportedly a high prevalence of schizophrenia among individuals with eating disorders [48]. CpG sites conforming the black module were enriched in morphine addiction (GABBR2, GABRP and $P D E 4 B)$, and polymorphisms of the $P D E 4 B$ gene have been associated with susceptibility to schizophrenia [49]. rs356971 and rs3129012 SNPs were correlated with the black module; these SNPs are associated with schizophrenia [50] and waist-hip index [27]. Furthermore, rs356971 and rs3129012 are associated with phenotypes related with the immunological system, hemoglobin concentration, white blood cells, and platelet count [51]. These SNPs are also associated with primary sclerosing cholangitis [52], autoimmune vitiligo [53], IgA deficiency [54], and systemic lupus erythematosus [55]. Immune-mediated mechanisms have been suggested in the development of EDs; an increased risk for autoimmune diseases in EDs has been reported [56,57]. Likewise, a locus in chromosome 12 was associated with anorexia nervosa, diabetes type 1, and autoimmune diseases [3].

Some modules could have comethylation of $\mathrm{CpG}$ sites which was not altered by a genetic effect in individuals diagnosed with an ED, like the turquoise and blue modules. These modules were enriched in pathways associated with the immunological system (Th1 and Th2 cell differentiation, TNF signaling pathway, focal adhesion). The same modules were also enriched in pathways related with development status. The construction of these modules could be influenced by the developmental stage of individuals in the sample, i.e., mainly teenagers [58]. One pathway is the GnRH (Gonadotropin Release Hormone) signaling pathway, which is activated at the beginning of pubertal development, and it depends on neuroendocrine signaling [59-61]. Another enriched pathway was associated with adiponectin (adiponectin/CaMKK/AMPK) [62,63]. Many authors suggest that adiponectin levels change with pubertal development [64,65]. Also, partial methylation of these modules suggests transcriptional activation of these pathways. The detection of these modules is more likely to be an effect of background epigenetic alterations and the cell development stage in the tissue (white blood cells) used for the analysis.

Our study has some limitations. Firstly, we note the absence of a control group. However, in this exploratory study, our primary aim was to detect comethylation modules in ED patients and assess the relationship between such modules and clinical phenotypes in ED. Second, we had a small sample with many variables evaluated. Although these conditions could affect the statistic power of our analysis, comethylation modules aggregate covarying CpGs and evaluate grouped CpGs, reducing the number of tests needed. Besides, WGCNA requires at least 20 samples to construct biologically meaningful modules [66]. 
Third, our data was from a sample made up of Mexican teenagers; therefore, our results should not be applied to all populations with EDs.

\section{Conclusions}

This is the first study integrating clinical features, genetic variants, and DNA methylation using comethylation network analysis in teenagers with ED. Our findings showed that two comethylation modules correlated with physical features as well as with SNPs previously associated with metabolic and psychiatric phenotypes. These data suggest that genetic variations could alter epigenetics, and that these perturbations could be reflected as variations in clinical features.

Supplementary Materials: The following are available online at https:/ / www.mdpi.com/article/10 .3390/nu13093210/s1, Table S1: CpG sites per chromosome and normalized value per chromosomic size, Table S2: Descriptive statistics of comethylation modules' beta values, Table S3: Functional enrichments of comethylation modules, Table S4: Characteristics of SNPs correlated with comethylation modules.

Author Contributions: Conceptualization, H.N. and A.D.G.-M.; methodology, G.A.N.-R. and I.E.J.R.; software, G.A.N.-R. and J.J.M.-M.; validation, I.E.J.-R. and C.A.T.-Z.; formal analysis, G.A.N.-R. and J.J.M.-M.; investigation, A.R.G., E.S., G.A.N.-R. and T.B.G.-C.; resources, H.N.; data curation, G.A.N.-R.; writing—original draft preparation, G.A.N.-R.; writing—review and editing, G.A.N.-R., J.J.M.-M., I.E.J.-R., T.B.G.-C., C.A.T.-Z., A.R.G., E.S., D.R.-R., A.D.G.-M. and H.N.; visualization, G.A.N.-R.; supervision, J.J.M.-M. and I.E.J.-R.; project administration, A.D.G.-M.; funding acquisition, H.N. All authors have read and agreed to the published version of the manuscript.

Funding: This research was funded by Fundación Gonzalo Río Arronte, grant number S591, and Instituto Nacional de Medicina Genómica (INMEGEN), grant number 06/2018/I.

Institutional Review Board Statement: This study was conducted according to the guidelines of the Declaration of Helsinki and approved by the Ethics Committees of the Psychiatric Hospital "Dr. Juan N. Navarro" (protocol code No. II3/01/0913; 11 October 2017), and National Institute of Genomic Medicine (INMEGEN) (protocol code No. 06/2018/I; June 2018).

Informed Consent Statement: Informed consent was obtained from all subjects involved in the study.

Data Availability Statement: The data presented in this study are available on request from the corresponding author, which were omitted due to privacy and ethical issues.

Acknowledgments: We want to acknowledge Microarray and Expression Unit of National Institute of Genomic Medicine for their technical support. Germán Alberto Nolasco-Rosales and David RuizRamos are students of Master of Biomedical Sciences degree in Juárez Autonomous University of Tabasco and were supported by CONACYT.

Conflicts of Interest: The authors declare no conflict of interest.

\section{References}

1. Bulik, C.M.; Blake, L.; Austin, J. Genetics of Eating Disorders: What the Clinician Needs to Know. Psychiatr. Clin. N. Am. 2019, 42, 59-73. [CrossRef]

2. Steiger, H.; Booij, L. Eating Disorders, Heredity and Environmental Activation: Getting Epigenetic Concepts into Practice. J. Clin. Med. 2020, 9, 1332. [CrossRef] [PubMed]

3. Duncan, L.; Yilmaz, Z.; Gaspar, H.; Walters, R.; Goldstein, J.; Anttila, V.; Bulik-Sullivan, B.; Ripke, S.; Thornton, L.; Hinney, A.; et al. Significant Locus and Metabolic Genetic Correlations Revealed in Genome-Wide Association Study of Anorexia Nervosa. Am. J. Psychiatry 2017, 174, 850-858. [CrossRef]

4. Yilmaz, Z.; Hardaway, J.A.; Bulik, C.M. Genetics and Epigenetics of Eating Disorders. Adv. Genom. Genet. 2015, 5, 131-150. [CrossRef]

5. Watson, H.J.; Yilmaz, Z.; Thornton, L.M.; Hübel, C.; Coleman, J.R.I.; Gaspar, H.A.; Bryois, J.; Hinney, A.; Leppä, V.M.; Mattheisen, M.; et al. Genome-wide association study identifies eight risk loci and implicates metabo-psychiatric origins for anorexia nervosa. Nat. Genet. 2019, 51, 1207-1214. [CrossRef] 
6. Munn-Chernoff, M.A.; Johnson, E.C.; Chou, Y.-L.; Coleman, J.R.I.; Thornton, L.M.; Walters, R.K.; Yilmaz, Z.; Baker, J.H.; Hübel, C.; Gordon, S.; et al. Shared genetic risk between eating disorder- and substance-use-related phenotypes: Evidence from genome-wide association studies. Addict. Biol. 2021, 26, e12880. [CrossRef]

7. Hübel, C.; Marzi, S.J.; Breen, G.; Bulik, C.M. Epigenetics in eating disorders: A systematic review. Mol. Psychiatry 2019, 24, 901-915. [CrossRef] [PubMed]

8. Rodríguez-López, M.L.; Martínez-Magaña, J.J.; Ruiz-Ramos, D.; García, A.R.; Gonzalez, L.; Tovilla-Zarate, C.A.; Sarmiento, E.; Juárez-Rojop, I.E.; Nicolini, H.; Gonzalez-Castro, T.B.; et al. Individuals Diagnosed with Binge-Eating Disorder Have DNA Hypomethylated Sites in Genes of the Metabolic System: A Pilot Study. Nutrients 2021, 13, 1413. [CrossRef]

9. Zhao, W.; Langfelder, P.; Fuller, T.; Dong, J.; Li, A.; Hovarth, S. Weighted Gene Coexpression Network Analysis: State of the Art. J. Biopharm. Stat. 2010, 20, 281-300. [CrossRef] [PubMed]

10. Lin, X.; Barton, S.; Holbrook, J.D. How to make DNA methylome wide association studies more powerful. Epigenomics 2016, 8, 1117-1129. [CrossRef]

11. Langfelder, P.; Horvath, S. WGCNA: An R package for weighted correlation network analysis. BMC Bioinform. 2008,9 , 559. [CrossRef]

12. APA. Diagnostic and Statistical Manual of Mental Disorders, 5th ed.; American Psychiatric Association: Arlington, VA, USA, 2013.

13. Yanovski, S.Z.; Marcus, M.D.; Wadden, T.A.; Walsh, B.T. The Questionnaire on Eating and Weight Patterns-5: An updated screening instrument for binge eating disorder. Int. J. Eat. Disord. 2015, 48, 259-261. [CrossRef]

14. Garner, D.M.; Olmsted, M.P.; Bohr, Y.; Garfinkel, P.E. The Eating Attitudes Test: Psychometric features and clinical correlates. Psychol. Med. 1982, 12, 871-878. [CrossRef]

15. Sheehan, D.V.; Sheehan, K.H.; Shytle, R.D.; Janavs, J.; Bannon, Y.; Rogers, J.E.; Milo, K.M.; Stock, S.L.; Wilkinson, B. Reliability and validity of the Mini International Neuropsychiatric Interview for Children and Adolescents (MINI-KID). J. Clin. Psychiatry 2010, 71, 313-326. [CrossRef]

16. Chang, C.C.; Chow, C.C.; Tellier, L.C.A.M.; Vattikuti, S.; Purcell, S.M.; Lee, J.J. Second-generation PLINK: Rising to the challenge of larger and richer datasets. GigaScience 2015, 4, s13742-015-0047-8. [CrossRef]

17. R Core Team. R: A Language and Environment for Statistical Computing; R Foundation for Statistical Computing: Vienna, Austria, 2020.

18. Morris, T.J.; Butcher, L.M.; Feber, A.; Teschendorff, A.E.; Chakravarthy, A.R.; Wojdacz, T.K.; Beck, S. ChAMP: 450k Chip Analysis Methylation Pipeline. Bioinformatics 2014, 30, 428-430. [CrossRef]

19. Teschendorff, A.E.; Marabita, F.; Lechner, M.; Bartlett, T.; Tegner, J.; Gomez-Cabrero, D.; Beck, S. A beta-mixture quantile normalization method for correcting probe design bias in Illumina Infinium $450 \mathrm{k}$ DNA methylation data. Bioinformatics 2013, 29, 189-196. [CrossRef] [PubMed]

20. Zhang, B.; Horvath, S. A General Framework for Weighted Gene Co-Expression Network Analysis. Stat. Appl. Genet. Mol. Biol. 2005, 4. [CrossRef] [PubMed]

21. Hansen, K.D. IlluminaHumanMethylationEPICanno.ilm10b4.hg19: Annotation for Illumina's EPIC Methylation Arrays, R Package Version 0.6.0. 2017.

22. Liao, Y.; Wang, J.; Jaehnig, E.J.; Shi, Z.; Zhang, B. WebGestalt 2019: Gene set analysis toolkit with revamped UIs and APIs. Nucleic Acids Res. 2019, 47, W199-W205. [CrossRef] [PubMed]

23. Kanehisa, M.; Goto, S. KEGG: Kyoto Encyclopedia of Genes and Genomes. Nucleic Acids Res. 2000, 28, 27-30. [CrossRef]

24. Watanabe, K.; Stringer, S.; Frei, O.; Umićević Mirkov, M.; de Leeuw, C.; Polderman, T.J.C.; van der Sluis, S.; Andreassen, O.A.; Neale, B.M.; Posthuma, D. A global overview of pleiotropy and genetic architecture in complex traits. Nat. Genet. 2019, 51, 1339-1348. [CrossRef]

25. Genis-Mendoza, A.D.; Martínez-Magaña, J.J.; Ruiz-Ramos, D.; Gonzalez-Covarrubias, V.; Tovilla-Zarate, C.A.; Narvaez, M.L.L.; Castro, T.B.G.; Juárez-Rojop, I.E.; Nicolini, H. Interaction of FTO rs9939609 and the native American-origin ABCA1 p.Arg230Cys with circulating leptin levels in Mexican adolescents diagnosed with eating disorders: Preliminary results. Psychiatry Res. 2020, 291, 113270. [CrossRef] [PubMed]

26. Hay, P. Current approach to eating disorders: A clinical update. Intern. Med. J. 2020, 50, 24-29. [CrossRef]

27. Pulit, S.L.; Stoneman, C.; Morris, A.P.; Wood, A.R.; Glastonbury, C.A.; Tyrrell, J.; Yengo, L.; Ferreira, T.; Marouli, E.; Ji, Y.; et al. Meta-analysis of genome-wide association studies for body fat distribution in 694649 individuals of European ancestry. Hum. Mol. Genet. 2019, 28, 166-174. [CrossRef] [PubMed]

28. Papaioannou, V.E. T-box genes in development: From hydra to humans. In International Review of Cytology; Academic Press: Cambridge, MA, USA, 2001; Volume 207, pp. 1-70.

29. Singh, M.K.; Petry, M.; Haenig, B.; Lescher, B.; Leitges, M.; Kispert, A. The T-box transcription factor Tbx15 is required for skeletal development. Mech. Dev. 2005, 122, 131-144. [CrossRef]

30. Lee, K.Y.; Singh, M.K.; Ussar, S.; Wetzel, P.; Hirshman, M.F.; Goodyear, L.J.; Kispert, A.; Kahn, C.R. Tbx15 controls skeletal muscle fibre-type determination and muscle metabolism. Nat. Commun. 2015, 6, 8054. [CrossRef]

31. Lee, K.Y.; Sharma, R.; Gase, G.; Ussar, S.; Li, Y.; Welch, L.; Berryman, D.E.; Kispert, A.; Bluher, M.; Kahn, C.R. Tbx15 Defines a Glycolytic Subpopulation and White Adipocyte Heterogeneity. Diabetes 2017, 66, 2822. [CrossRef] [PubMed]

32. Sun, P.; Zhang, D.; Huang, H.; Yu, Y.; Yang, Z.; Niu, Y.; Liu, J. MicroRNA-1225-5p acts as a tumor-suppressor in laryngeal cancer via targeting CDC14B. Biol. Chem. 2019, 400, 237-246. [CrossRef] [PubMed] 
33. Cohen, P.; Levy, J.D.; Zhang, Y.; Frontini, A.; Kolodin, D.P.; Svensson, K.J.; Lo, J.C.; Zeng, X.; Ye, L.; Khandekar, M.J.; et al. Ablation of PRDM16 and Beige Adipose Causes Metabolic Dysfunction and a Subcutaneous to Visceral Fat Switch. Cell 2014, 156, 304-316. [CrossRef]

34. Arensdorf, A.M.; Dillard, M.E.; Menke, J.M.; Frank, M.W.; Rock, C.O.; Ogden, S.K. Sonic Hedgehog Activates Phospholipase A2 to Enhance Smoothened Ciliary Translocation. Cell Rep. 2017, 19, 2074-2087. [CrossRef]

35. Iyer, A.; Lim, J.; Poudyal, H.; Reid, R.C.; Suen, J.Y.; Webster, J.; Prins, J.B.; Whitehead, J.P.; Fairlie, D.P.; Brown, L. An Inhibitor of Phospholipase $\mathrm{A}^{2}$; Group IIA Modulates Adipocyte Signaling and Protects Against Diet-Induced Metabolic Syndrome in Rats. Diabetes 2012, 61, 2320. [CrossRef] [PubMed]

36. Kuefner, M.S.; Deng, X.; Stephenson, E.J.; Pham, K.; Park, E.A. Secretory phospholipase A2 group IIA enhances the metabolic rate and increases glucose utilization in response to thyroid hormone. FASEB J. 2019, 33, 738-749. [CrossRef] [PubMed]

37. Sato, H.; Taketomi, Y.; Ushida, A.; Isogai, Y.; Kojima, T.; Hirabayashi, T.; Miki, Y.; Yamamoto, K.; Nishito, Y.; Kobayashi, T.; et al. The Adipocyte-Inducible Secreted Phospholipases PLA2G5 and PLA2G2E Play Distinct Roles in Obesity. Cell Metab. 2014, 20, 119-132. [CrossRef]

38. Shin, S.-Y.; Fauman, E.B.; Petersen, A.-K.; Krumsiek, J.; Santos, R.; Huang, J.; Arnold, M.; Erte, I.; Forgetta, V.; Yang, T.-P.; et al. An atlas of genetic influences on human blood metabolites. Nat. Genet. 2014, 46, 543-550. [CrossRef]

39. Coan, P.M.; Hummel, O.; Garcia Diaz, A.; Barrier, M.; Alfazema, N.; Norsworthy, P.J.; Pravenec, M.; Petretto, E.; Hübner, N.; Aitman, T.J. Genetic, physiological and comparative genomic studies of hypertension and insulin resistance in the spontaneously hypertensive rat. Dis. Models Mech. 2017, 10, 297-306. [CrossRef] [PubMed]

40. Yengo, L.; Sidorenko, J.; Kemper, K.E.; Zheng, Z.; Wood, A.R.; Weedon, M.N.; Frayling, T.M.; Hirschhorn, J.; Yang, J.; Visscher, P.M.; et al. Meta-analysis of genome-wide association studies for height and body mass index in $~ 700000$ individuals of European ancestry. Hum. Mol. Genet. 2018, 27, 3641-3649. [CrossRef]

41. Belanger, K.; Nutter, C.A.; Li, J.; Tasnim, S.; Liu, P.; Yu, P.; Kuyumcu-Martinez, M.N. CELF1 contributes to aberrant alternative splicing patterns in the type 1 diabetic heart. Biochem. Biophys. Res. Commun. 2018, 503, 3205-3211. [CrossRef]

42. Chang, K.-T.; Cheng, C.-F.; King, P.-C.; Liu, S.-Y.; Wang, G.-S. CELF1 Mediates Connexin 43 mRNA Degradation in Dilated Cardiomyopathy. Circ. Res. 2017, 121, 1140-1152. [CrossRef]

43. Fang, Y.; Tao, Y.; Zhou, H.; Lai, H. Promoting role of circ-Jarid2/miR-129-5p/Celf1 axis in cardiac hypertrophy. Gene Ther. 2020, 27, 1-11. [CrossRef]

44. Sciarretta, S.; Forte, M.; Frati, G.; Sadoshima, J. New Insights into the Role of mTOR Signaling in the Cardiovascular System. Circ. Res. 2018, 122, 489-505. [CrossRef]

45. Sciarretta, S.; Volpe, M.; Sadoshima, J. Mammalian Target of Rapamycin Signaling in Cardiac Physiology and Disease. Circ. Res. 2014, 114, 549-564. [CrossRef]

46. Fayssoil, A.; Melchior, J.C.; Hanachi, M. Heart and anorexia nervosa. Heart Fail. Rev. 2021, 26, 65-70. [CrossRef]

47. Westmoreland, P.; Krantz, M.J.; Mehler, P.S. Medical Complications of Anorexia Nervosa and Bulimia. Am. J. Med. 2016, 129, 30-37. [CrossRef] [PubMed]

48. Kouidrat, Y.; Amad, A.; Lalau, J.-D.; Loas, G. Eating Disorders in Schizophrenia: Implications for Research and Management. Schizophr. Res. Treat. 2014, 2014, 791573. [CrossRef] [PubMed]

49. Feng, Y.; Cheng, D.; Zhang, C.; Li, Y.; Zhang, Z.; Wang, J.; Shi, Y. Association of PDE4B Polymorphisms with Susceptibility to Schizophrenia: A Meta-Analysis of Case-Control Studies. PLoS ONE 2016, 11, e0147092. [CrossRef] [PubMed]

50. Pardiñas, A.F.; Holmans, P.; Pocklington, A.J.; Escott-Price, V.; Ripke, S.; Carrera, N.; Legge, S.E.; Bishop, S.; Cameron, D.; Hamshere, M.L.; et al. Common schizophrenia alleles are enriched in mutation-intolerant genes and in regions under strong background selection. Nat. Genet. 2018, 50, 381-389. [CrossRef]

51. Astle, W.J.; Elding, H.; Jiang, T.; Allen, D.; Ruklisa, D.; Mann, A.L.; Mead, D.; Bouman, H.; Riveros-Mckay, F.; Kostadima, M.A.; et al. The Allelic Landscape of Human Blood Cell Trait Variation and Links to Common Complex Disease. Cell 2016, 167, 1415-1429.e1419. [CrossRef] [PubMed]

52. Ji, S.-G.; Juran, B.D.; Mucha, S.; Folseraas, T.; Jostins, L.; Melum, E.; Kumasaka, N.; Atkinson, E.J.; Schlicht, E.M.; Liu, J.Z.; et al. Genome-wide association study of primary sclerosing cholangitis identifies new risk loci and quantifies the genetic relationship with inflammatory bowel disease. Nat. Genet. 2017, 49, 269-273. [CrossRef] [PubMed]

53. Jin, Y.; Andersen, G.; Yorgov, D.; Ferrara, T.M.; Ben, S.; Brownson, K.M.; Holland, P.J.; Birlea, S.A.; Siebert, J.; Hartmann, A.; et al. Genome-wide association studies of autoimmune vitiligo identify 23 new risk loci and highlight key pathways and regulatory variants. Nat. Genet. 2016, 48, 1418-1424. [CrossRef] [PubMed]

54. Bronson, P.G.; Chang, D.; Bhangale, T.; Seldin, M.F.; Ortmann, W.; Ferreira, R.C.; Urcelay, E.; Pereira, L.F.; Martin, J.; Plebani, A.; et al. Common variants at PVT1, ATG13-AMBRA1, AHI1 and CLEC16A are associated with selective IgA deficiency. Nat. Genet. 2016, 48, 1425-1429. [CrossRef]

55. Julià, A.; López-Longo, F.J.; Pérez Venegas, J.J.; Bonàs-Guarch, S.; Olivé, À.; Andreu, J.L.; Aguirre-Zamorano, M.Á.; Vela, P.; Nolla, J.M.; de la Fuente, J.L.M.; et al. Genome-wide association study meta-analysis identifies five new loci for systemic lupus erythematosus. Arthritis Res. Ther. 2018, 20, 100. [CrossRef] [PubMed]

56. Raevuori, A.; Haukka, J.; Vaarala, O.; Suvisaari, J.M.; Gissler, M.; Grainger, M.; Linna, M.S.; Suokas, J.T. The Increased Risk for Autoimmune Diseases in Patients with Eating Disorders. PLoS ONE 2014, 9, e104845. [CrossRef] 
57. Zerwas, S.; Larsen, J.T.; Petersen, L.; Thornton, L.M.; Quaranta, M.; Koch, S.V.; Pisetsky, D.; Mortensen, P.B.; Bulik, C.M. Eating Disorders, Autoimmune, and Autoinflammatory Disease. Pediatrics 2017, 140, e20162089. [CrossRef] [PubMed]

58. Almstrup, K.; Lindhardt Johansen, M.; Busch, A.S.; Hagen, C.P.; Nielsen, J.E.; Petersen, J.H.; Juul, A. Pubertal development in healthy children is mirrored by DNA methylation patterns in peripheral blood. Sci. Rep. 2016, 6, 28657. [CrossRef]

59. Abreu, A.P.; Kaiser, U.B. Pubertal development and regulation. Lancet Diabetes Endocrinol. 2016, 4, 254-264. [CrossRef]

60. Herbison, A.E. Control of puberty onset and fertility by gonadotropin-releasing hormone neurons. Nat. Rev. Endocrinol. 2016, 12, 452-466. [CrossRef]

61. Livadas, S.; Chrousos, G.P. Control of the onset of puberty. Curr. Opin. Pediatrics 2016, 28, 551-558. [CrossRef]

62. Fang, H.; Judd, R.L. Adiponectin Regulation and Function. Compr. Physiol. 2018, 8, 1031-1063. [CrossRef]

63. Wang, Z.V.; Scherer, P.E. Adiponectin, the past two decades. J. Mol. Cell Biol. 2016, 8, 93-100. [CrossRef] [PubMed]

64. Sitticharoon, C.; Sukharomana, M.; Likitmaskul, S.; Churintaraphan, M.; Maikaew, P. Increased high molecular weight adiponectin, but decreased total adiponectin and kisspeptin, in central precocious puberty compared with aged-matched prepubertal girls. Reprod. Fertil. Dev. 2017, 29, 2466-2478. [CrossRef]

65. Woo, J.G.; Dolan, L.M.; Daniels, S.R.; Goodman, E.; Martin, L.J. Adolescent Sex Differences in Adiponectin Are Conditional on Pubertal Development and Adiposity. Obes. Res. 2005, 13, 2095-2101. [CrossRef] [PubMed]

66. Langfelder, P.; Horvath, S. WGCNA Package FAQ. Available online: https://horvath.genetics.ucla.edu/html/CoexpressionNetwork/ Rpackages/WGCNA/faq.html (accessed on 24 August 2021). 